\title{
A Weighted Least Squares Approach to Precoding with Pilots for MIMO-OFDM
}

\author{
Tarkesh Pande, David J. Love, and James V. Krogmeier \\ School of Electrical and Computer Engineering \\ Purdue University \\ West Lafavette, IN 47907 \\ \{pande, djlove, jvk\}@ecn.purdue.edu
}

December 14, 2005

\begin{abstract}
Next generation wireless systems are expected to leverage multiple antenna, commonly called multipleinput multiple-output (MIMO), technology transmitting over broadband channels via orthogonal frequency division multiplexing (OFDM). It is now well-known that MIMO systems can obtain error rate and capacity improvements by adapting the transmitter to the current channel conditions. Linear spatial precoding is a popular technique for channel adaptation where the transmitted space-time signal for each subcarrier is multiplied by a precoding matrix before transmission. Spatial precoding is complicated, however, in OFDM because channel information is often only known for a small number of pilot tones. In this paper, we present a subspace interpolation method for designing spatial precoders for all subcarriers by using the precoders known on pilot tones. The non-pilot precoders are solutions to a weighted least squares design on the Grassmann manifold. Simulation results show performance benefits over existing MIMO-OFDM techniques.
\end{abstract}

Index Terms-Closed-loop, Interpolation, Limited feedback, MIMO systems, Orthogonal frequency division multiplexing, Subspace coding.

Permission to publish abstract separately granted.

This work was supported in part by the SBC Foundation, NSF under Grant CCF0513916 and the Indiana Twenty First Century Research and Technology Fund.

\section{Introduction}

Recent work has shown that multiple antenna systems (often called multiple-input multiple-output (MIMO) systems) exploiting channel knowledge at the transmitter, commonly called closed-loop systems, can obtain large benefits in error rate reduction and capacity improvements compared to non-adaptive systems. In narrowband systems that experience flat-fading, methods have been devised allowing both time division duplexing (TDD) and frequency division duplexing (FDD) to efficiently use channel knowledge. Because of the substantial data rate requirements, next generation communication systems will transmit over large bandwidths with signals that experience frequency-selective fading. One effective method for dealing with frequency-selectivity is orthogonal frequency division multiplexing (OFDM). OFDM constructs a broadband 
signal using an orthogonal transformation applied to a multitude of narrowband signals. The end result is that instead of one high-rate multi-tap channel we are left with a number of lower-rate single-tap channels.

Applying closed-loop techniques is challenging because of the number of different channels corresponding to different subcarriers. Channel estimation for OFDM is commonly done using pilots [1]. The general idea is for the OFDM symbol to interlace $K$ pilots tones within the $N$ subcarriers. This means that each OFDM symbol still has $N-K$ subcarriers transmitting user data. Because the data conveyed on the pilot tones is predetermined, the transmitted symbol can be divided out of the received signal to yield a flat-fading channel estimate at each pilot. Thus, the receiver will have knowledge of the $K$ channels corresponding to the pilots. Common OFDM channel estimation techniques use interpolation methods on the channels obtained from the $K$ pilots to estimate the channel for all $N$ subcarriers. Example interpolators include minimum mean squared error [2] and linear interpolation [3]-[6].

We are interested not in channel estimation but rather in adapting the transmitted signal to the current channel conditions. Linear precoding [7]-[9] is a practical solution that adapts the transmitted space-time signal (e.g., spatial multiplexing, orthogonal space-time block coding, etc.) to the channel by multiplying the signal by a matrix before transmission. The intuition here is that the matrix directs the transmitted spacetime signal towards the "good" directions of the channel while avoiding the "bad". In TDD narrowband systems, precoders can be designed at the transmitter for the forward-link channel using the channel estimate on the reverse-link. The transmitter in an FDD narrowband system, however, does not have knowledge of the forward-link channel matrix. This can be overcome through the use of feedback from the receiver to the transmitter [10]-[18].

In this paper, we address the general problem of designing precoding matrices for all the tones given only the precoding matrices for the pilot tones. Just as in $[12],[13],[15],[16]$, we view the precoders as points in the Grassmann manifold. The interpolator solves a weighted least squares problem on the Grassmann manifold. Thus, the interpolation is done on subspaces rather than matrices using a subspace, rather than Euclidean, distance. Our interpolators can be based on any number of pilots and can be simply implemented 
using common techniques from linear algebra. Specifically, we show that this interpolation relates to finding the principle components of a matrix obtained by combining the weighted generator matrices of the precoder subspaces.

This is a different approach than taken in [19]-[21] where the precoders are designed using a phase invariance weighting of the nearest two pilots meant to maximize some condition. Our technique can include any number of pilot tones. In addition, we do not require feedback of a unitary subspace rotation matrix as in [21]. This reduction in feedback can be significant when the number of pilots is large. This problem has practical significance particularly with application to next generation wireless local area networks (WLANs) and wireless metropolitan access.

The organization of this paper is as follows. Section 2 gives a general overview of precoding for MIMOOFDM. We propose an interpolation scheme that uses the precoders obtained from pilot subcarriers to design precoders for all $N$ subcarriers in Section 3. Section 4 gives simulation results. We conclude in Section 5 .

\section{Precoding Systems}

Consider an $M_{t}$ transmit antenna and $M_{r}$ receive antenna MIMO-OFDM system transmitting with $N$ subcarriers. We will assume that the signal experiences an $L$-tap frequency selective channel in time given by ${ }^{1}$

$$
\mathbf{G}[i]=\sum_{l=0}^{L-1} \mathbf{G}_{l} \delta[i-l]
$$

where $\mathbf{G}_{l} \in \mathbb{C}^{M_{r} \times M_{t}}$ for all $l$. We will assume that $\mathbf{G}_{l}$ has independent and identically $\mathcal{C N}(0,1)$ distributed entries and that $\mathbf{G}_{l_{1}}$ is independent of $\mathbf{G}_{l_{2}}$ for all $0 \leq l_{1}<l_{2}<L$.

We will deal with the signal design and analysis in the frequency domain using an $N$-point inverse fast Fourier transformation (IFFT) at the transmitter and an $N$-point fast Fourier transformation (FFT) at the

\footnotetext{
${ }^{1}$ We use $j=\sqrt{-1}, \delta[\cdot]$ to denote the Kronecker delta function, ${ }^{*}$ to denote matrix conjugate transposition, ${ }^{+}$to denote the pseudo-inverse, $E[\cdot]$ to denote expectation, $\mathbb{C}^{k \times l}$ to denote the vector space of $k \times l$ complex matrices, $\mathbf{I}_{M}$ to denote the $M \times M$ identity matrix, $\mathcal{C N}(0,1)$ to denote a complex Gaussian random variable with zero mean and unit variance, $\lambda_{i}\{\mathbf{A}\}$ to denote the $i^{\text {th }}$ singular value of $\mathbf{A},|\cdot|$ to denote the absolute value, $\operatorname{tr}(\cdot)$ to denote the trace of a matrix, $\|\cdot\|_{2}$ to denote the matrix two-norm, $\|\cdot\|_{F}$ to denote the matrix Frobenius norm, $\mathcal{U}\left(M_{t}, M\right)$ to denote the set of $M_{t} \times M$ matrices with orthonormal columns, $\operatorname{card}(\cdot)$ to denote the cardinality of a set, and the combinatorial function $\left(\begin{array}{l}n \\ k\end{array}\right)=n ! /[k !(n-k) !]$.
} 
receiver. In addition, we will assume that the length of the cyclic prefix is greater than the intersymbol interference (ISI) length introduced by the channel. After removing the cyclic prefix at the receiver the MIMO frequency-domain channel for the $n^{\text {th }}$ subcarrier (where $n=0,1, \ldots, N-1$ ) is given by

$$
\mathbf{H}_{n}=\frac{1}{\sqrt{N}} \sum_{l=0}^{L-1} \mathbf{G}_{l} \exp \left(-j \frac{2 \pi n l}{N}\right) .
$$

This allows us to write the input-output relationship for the $n^{\text {th }}$ tone as

$$
\mathbf{Y}_{n}=\mathbf{H}_{n} \mathbf{X}_{n}+\mathbf{N}_{n}
$$

where $\mathbf{N}_{n}$ is an $M_{r} \times T$ matrix with $\mathcal{C N}(0,1)$ entries, $\mathbf{X}_{n}$ is an $M_{t} \times T$ transmitted matrix with $\operatorname{tr}\left(E\left[\mathbf{X}_{n}^{*} \mathbf{X}_{n}\right]\right)=$ $T \rho, \rho$ is the signal-to-noise ratio (SNR), and $T$ is the number of channel uses involved in transmitting one space-time signal. Examples of $T$ include $T=1$ for spatial multiplexing [22], [23], $T=2$ for a two-antenna Alamouti code [24], and $T=1$ for beamforming (i.e. one-dimensional precoding) [22].

The transmitted signal $\mathbf{X}_{n}$ can be classified into two cases: open-loop and closed-loop. In an open-loop system, the signal $\mathbf{X}_{n}$ is designed independently of the channel $\mathbf{H}_{n}$. In a closed-loop system, the signal $\mathbf{X}_{n}$ is designed as a function of $\mathbf{H}_{n}$. We will assume that the MIMO-OFDM system uses a special kind of closed-loop signaling known as linear precoding [7]-[9]. In linear precoding, the transmitted matrix can be decomposed as

$$
\mathbf{X}_{n}=\mathbf{F}_{n} \mathbf{S}_{n}
$$

where $\mathbf{F}_{n}$ is an $M_{t} \times M$ (with $1 \leq M \leq M_{t}$ ) precoding matrix and $\mathbf{S}_{n}$ is an $M \times T$ space-time signal. The space-time signal could be generated with an $M$-dimensional spatial multiplexing encoder $\left(\mathbf{S}_{n}=\mathbf{s}_{n} \in \mathbb{C}^{M \times 1}\right)$ [23], a single-dimensional modulator $\left(\mathbf{S}_{n}=s_{n} \in \mathbb{C}^{1 \times 1}\right)$ [22], or an $M$ dimensional orthogonal space-time block code [25] $\left(\mathbf{S}_{n} \in \mathbb{C}^{M \times T}\right)$. To constrain the peak transmitted power, we will assume that $\mathbf{F}_{n} \in \mathcal{U}\left(M_{t}, M\right)$ for all subcarriers . This approach is a natural extension of the work in antenna subset selection space-time signaling (see for example [26]-[28]). This kind of channel orthogonalization precoding has been used for beamforming [10], [12], [13], precoded orthogonal space-time block codes [15], and precoded spatial multiplexing [7], [16]. 
The optimal unquantized precoders for the above space-time signaling schemes can be determined from the selection criteria being considered. Precoding criteria can generally be divided into two categories i) minimizing the error rate based on the receiver for the space-time signaling scheme ii) maximizing the mutual information for the precoded channel.

\section{i) Minimizing Error Rate}

a) Beamforming: An equivalent objective to minimizing the average probability of symbol error is to maximize the post processed SNR [29]. For beamforming systems the SNR $\gamma_{n}$ seen at the receiver for each subcarrier after combining is

$$
\gamma_{n}=\frac{\rho\left|\mathbf{F}_{n}^{*} \mathbf{H}_{n}^{*} \mathbf{H}_{n} \mathbf{F}_{n}\right|^{2}}{\left\|\mathbf{F}_{n}^{*} \mathbf{H}_{n}^{*}\right\|_{2}^{2}}
$$

In this case the precoder vector $\mathbf{F}_{\text {opt }}$ that maximizes (5) is the right singular vector of $\mathbf{H}_{n}$ corresponding to the largest singular value of $\mathbf{H}_{n}$ [12]. Note that $e^{j \phi} \mathbf{F}_{\text {opt }}$ for an arbitrary $\phi$ also maximizes (5) implying that the optimal precoder is not unique. In fact, all vectors with the same column space provide the same performance.

b) Spatial Multiplexing: For spatial multiplexing systems, commonly used receiver architectures do either i) maximum likelihood (ML) decoding, ii) minimize the minimum mean square error (MMSE) or iii) zero-forcing (ZF) . The ML receiver while providing the best performance has the highest computational complexity. MMSE and ZF decoders are linear receivers whereby a linear transformation $\mathbf{C}$, an $M \times M_{r}$ matrix, is first applied to the received signal $\mathbf{Y}_{n}$. This is followed by an operation $\mathbf{Q}(\cdot)$ which does single dimension $\mathrm{ML}$ decoding on each substream to get the estimate of the transmitted space-time vector signal i.e., $\widehat{\mathbf{S}}_{n}=\mathbf{Q}\left(\mathbf{C Y}_{n}\right)$. For MMSE decoding, $\mathbf{C}=\left[\mathbf{F}_{n}^{*} \mathbf{H}_{n}^{*} \mathbf{H}_{n} \mathbf{F}_{n}+\left(M N_{0} / \mathcal{E}_{s}\right) \mathbf{I}_{M}\right]^{-1} \mathbf{F}_{n}^{*} \mathbf{H}_{n}^{*}$ while the ZF receiver has the form $\mathbf{C}=\left(\mathbf{H}_{n} \mathbf{F}_{n}\right)^{+}$. We now review the precoding selection criterion for ZF systems. Precoding for ML and MMSE receivers can be found in [16] and references therein.

In [30], it is shown that the union bound of the vector symbol error performance for a ZF system 
depends on the substream with minimum SNR. For this case

$$
S N R_{\min }^{(Z F)} \geq \lambda_{\min }^{2}\left\{\mathbf{H}_{n} \mathbf{F}_{n}\right\} \frac{\mathcal{E}_{\mathbf{s}}}{M N_{0}}
$$

where $\lambda_{\min }\left\{\mathbf{H}_{n} \mathbf{F}_{n}\right\}$ is the minimum singular value of $\mathbf{H}_{n} \mathbf{F}_{n}$. The optimum unquantized precoder maximizes (6) which in turn minimizes the vector symbol error probability. Let the singular value decomposition of $\mathbf{H}_{n}$ be given by

$$
\mathbf{H}_{n}=\mathbf{V}_{L} \boldsymbol{\Sigma} \mathbf{V}_{R}^{*}
$$

where $\mathbf{V}_{L} \in \mathcal{U}\left(M_{r}, M_{r}\right), \mathbf{V}_{R} \in \mathcal{U}\left(M_{t}, M_{t}\right)$ and $\boldsymbol{\Sigma}$ is an $M_{r} \times M_{t}$ diagonal matrix containing the singular values of $\mathbf{H}_{n}$. In [16], it is shown that the optimal precoder matrix is $\mathbf{F}_{\text {opt }}=\overline{\mathbf{V}}_{R}$ where $\overline{\mathbf{V}}_{R}$ is the matrix constructed from the first $M$ columns of $\mathbf{V}_{R}$. The optimal precoder matrix is not unique as $\mathbf{F}_{\text {opt }} \mathbf{U}, \mathbf{U} \in \mathcal{U}(M, M)$ provides performance identical to that of $\mathbf{F}_{\text {opt }}$. This can be easily seen because [31]

$$
\lambda_{\min }\left\{\mathbf{H}_{n} \mathbf{F}_{\text {opt }} \mathbf{U}\right\}=\lambda_{M}\left\{\mathbf{V}_{L} \boldsymbol{\Sigma} \mathbf{V}_{R}^{*} \mathbf{F}_{\text {opt }} \mathbf{U}\right\} \leq \lambda_{M}\left(\mathbf{H}_{n}\right)
$$

where $\lambda_{M}\left(\mathbf{H}_{n}\right)$ is the $M^{\text {th }}$ singular value of $\mathbf{H}_{n}$. In (8) the upper bound is achieved when $\mathbf{F}_{\text {opt }}=\overline{\mathbf{V}}_{R}$. The minimum singular value is invariant to right multiplication by a unitary matrix. Therefore, all matrices that generate the same column space give the same minimum singular value performance.

c) Precoded Orthogonal Space-Time Block Coding (OSTBC): For precoded OSTBC, the probability of symbol error (SER) given channel knowledge $\mathbf{H}_{n}$ when using an ML detector can be written as $[32]$

$$
\operatorname{Pr}\left(E R R O R \mid \mathbf{H}_{n}\right) \leq \exp \left(-\gamma\left\|\mathbf{H}_{n} \mathbf{F}_{n}\right\|_{F}^{2}\right)
$$

where $\gamma$ is a function that depends on $M, \rho$ and $\mathbf{S}_{n}$. If $\gamma$ is fixed, minimizing SER corresponds to maximizing $\left\|\mathbf{H}_{n} \mathbf{F}_{n}\right\|_{F}^{2}$. In [15] it is shown that the optimal precoder corresponds to $\mathbf{F}_{\text {opt }}=\overline{\mathbf{V}}_{R}$. 
Again, precoder performance is invariant to right multiplication by a unitary matrix as

$$
\left\|\mathbf{H}_{n} \mathbf{F}_{n} \mathbf{U}\right\|_{F}^{2}=\operatorname{tr}\left(\mathbf{U}^{*} \mathbf{F}_{n}^{*} \mathbf{H}_{n}^{*} \mathbf{H}_{n} \mathbf{F}_{n} \mathbf{U}\right)=\operatorname{tr}\left(\mathbf{F}_{n}^{*} \mathbf{H}_{n}^{*} \mathbf{H}_{n} \mathbf{F}_{n}\right)=\left\|\mathbf{H}_{n} \mathbf{F}_{n}\right\|_{F}^{2}
$$

\section{ii) Capacity}

As opposed to minimizing symbol error rates, an alternative criteria for precoding is to determine $\mathbf{F}_{n}$ that maximizes the capacity of the channel [27], [33]. The uninformed transmitter capacity or mutual information $I\left(\mathbf{F}_{n}\right)$ assuming an uncorrelated complex Gaussian source given $\mathbf{H}_{n}$ and a fixed $\mathbf{F}_{n}$ is

$$
I\left(\mathbf{F}_{n}\right)=\log _{2} \operatorname{det}\left(\mathbf{I}_{M}+\frac{\mathcal{E}_{s}}{M N_{0}} \mathbf{F}_{n}^{*} \mathbf{H}_{n}^{*} \mathbf{H}_{n} \mathbf{F}_{n}\right)
$$

The precoder maximizing the above is also $\mathbf{F}_{\text {opt }}=\overline{\mathbf{V}}_{R}[16]$. This precoding criteria applies to all the above space-time signaling schemes. Finally, it is worth noting that precoder performance depends only on its column space as maximizing $I\left(\mathbf{F}_{n} \mathbf{U}\right)$ for $\mathbf{U} \in \mathcal{U}(M, M)$ is equivalent to maximizing $\operatorname{det}\left(\mathbf{U}^{*}\left(\mathbf{I}_{M}+\frac{\mathcal{E}_{s}}{M N_{0}} \mathbf{F}_{n}^{*} \mathbf{H}_{n}^{*} \mathbf{H}_{n} \mathbf{F}_{n}\right) \mathbf{U}\right)$ which is equivalent to maximizing $\operatorname{det}\left(\mathbf{I}_{M}+\frac{\mathcal{E}_{s}}{M N_{0}} \mathbf{F}_{n}^{*} \mathbf{H}_{n}^{*} \mathbf{H}_{n} \mathbf{F}_{n}\right)$. For MIMO-OFDM systems the linear precoding matrix $\mathbf{F}_{n}$ must be designed as a function of $\mathbf{H}_{n}$ for every subcarrier. In this paper, we will study the use of pilots that are interspersed throughout the OFDM symbol. We will assume that there are $K$ pilots transmitting known data. The $k^{\text {th }}$ pilot (with $k=0,1, \ldots, K-1$ ) is located at subcarrier $k \frac{N}{K}$. This is a comb-type tone arrangement and has been studied in [1].

Mathematically, the pilots correspond to

$$
\mathbf{Y}^{(k)}=\mathbf{H}^{(k)} \boldsymbol{\Xi}^{(k)}+\mathbf{N}^{(k)}
$$

for the $k^{\text {th }}$ pilot. The matrix $\boldsymbol{\Xi}^{(k)}$ is some sort of training space-time signal that is known to both the transmitter and the receiver and allows the receiver to solve for $\mathbf{H}^{(k)}$ for $0 \leq k \leq K-1$.

In TDD, perfect channel reciprocity would allow the transmitter to also know $\mathbf{H}^{(k)}$ for $0 \leq k \leq K-1$. In FDD, the transmitter has no knowledge of the channels corresponding to the forward-link pilots. Thus, some form of feedback must be used. We will consider the idea of using the narrowband feedback techniques developed in [10]-[13], [15]-[18] for each pilot subcarrier in the OFDM symbol. The receiver will use its 
knowledge of $\mathbf{H}^{(k)}$ for $0 \leq k \leq K-1$ to design precoders $\mathbf{F}^{(k)}$ for each pilot. The idea is to restrict each $\mathbf{F}^{(k)}$ to lie in a codebook $\mathcal{F}=\left\{\mathbf{F}_{1}, \mathbf{F}_{2}, \ldots, \mathbf{F}_{2^{B}}\right\}$. The codebook is known to both the transmitter and the receiver. Because of this, a total of $K \cdot B$ bits of feedback can be sent from the receiver to the transmitter to convey the $K$ precoders for the pilots that were chosen from the codebook.

Because subcarrier numbers can possibly number in the hundreds, it is impractical for an FDD system to feedback a total of $N \cdot B$ bits of feedback to design $\mathbf{F}_{n}$ for $0 \leq n \leq N-1$. For this reason, it is of utmost importance to find efficient methods for determining all $\mathbf{F}_{n}$ precoders from $\mathbf{F}^{(1)}, \mathbf{F}^{(2)}, \cdots, \mathbf{F}^{(K)}$.

\section{Interpolation Precoding}

We will discuss the interpretation of precoders as points in the Grassmann manifold before presenting the interpolator.

\subsection{Grassmannian Precoding}

As described in the previous section, the precoder $\mathbf{F}_{n}$ is usually chosen with respect to some criterion such as i) maximizing the minimum singular vector of $\mathbf{H}_{n} \mathbf{F}_{n}$, ii) maximizing the capacity of the effective channel $\mathbf{H}_{n} \mathbf{F}_{n}$, iii) minimizing the mean squared error (with a minimum mean squared error receiver) when transmitting over $\mathbf{H}_{n} \mathbf{F}_{n}$, or iv) maximizing the Frobenius norm of $\mathbf{H}_{n} \mathbf{F}_{n}$. All of these criteria are invariant to multiplication of $\mathbf{F}_{n}$ by an $M \times M$ unitary matrix $\mathbf{U} \in \mathcal{U}(M, M)$. This means that, from the perspective of these criteria, $\mathbf{F}_{n} \mathbf{U}$ provides performance identical to $\mathbf{F}_{n}$. Note that this means the performance is dependent only on the column space of $\mathbf{F}_{n}$. This kind of invariance was used in [12], [13], [15], [16] to significantly reduce the quantization problem of designing $\mathcal{F}$ for use in FDD systems.

Because of this invariance, we will view each precoder $\mathbf{F}_{n}$ as a subspace rather than a matrix. The set of all $M$-dimensional subspaces of $\mathbb{C}^{M_{t}}$ is known as the Grassmann manifold and denoted by $\mathcal{G}\left(M_{t}, M\right)$. Distances can be defined on the Grassmann manifold just as on standard Euclidean spaces [34]. The chordal distance between subspace $\mathcal{P}_{1}$ and $\mathcal{P}_{2}$ with corresponding orthogonal basis matrices $\mathbf{F}_{\mathcal{P}_{1}}$ and $\mathbf{F}_{\mathcal{P}_{2}}$ (i.e., 
$\left.\mathbf{F}_{\mathcal{P}_{1}}, \mathbf{F}_{\mathcal{P}_{2}} \in \mathcal{U}\left(M_{t}, M\right)\right)$ is given by

$$
d\left(\mathbf{F}_{\mathcal{P}_{1}}, \mathbf{F}_{\mathcal{P}_{2}}\right)=\frac{1}{\sqrt{2}}\left\|\mathbf{F}_{\mathcal{P}_{1}} \mathbf{F}_{\mathcal{P}_{1}}^{*}-\mathbf{F}_{\mathcal{P}_{2}} \mathbf{F}_{\mathcal{P}_{2}}^{*}\right\|_{F}
$$

where $\|\cdot\|_{F}$ denotes the Frobenius norm. While this distance is written as a function of the basis matrix, the distance is only a function of the subspaces since $\mathbf{F} \mathbf{U} \mathbf{U}^{*} \mathbf{F}^{*}=\mathbf{F} \mathbf{F}^{*}$ when $\mathbf{F} \in \mathcal{U}\left(M_{t}, M\right)$ and $\mathbf{U} \in \mathcal{U}(M, M)$.

Two subspaces in $\mathcal{G}\left(M_{t}, M\right)$ can be oriented in relation to each other using the $M$ principal angles $\phi_{1}, \ldots, \phi_{M}$ between the subspaces. In the $M=1$ case, there is only one principal angle and it is given by

$\arccos \left(\left|\mathbf{f}_{\mathcal{P}_{1}}^{*} \mathbf{f}_{\mathcal{P}_{2}}\right|\right)$ where $\mathbf{f}_{\mathcal{P}_{i}}$ is the unit vector that generates the one-dimensional subspace (or line) $\mathcal{P}_{i}$. More generally,

$$
\sum_{i=1}^{M} \cos ^{2}\left(\phi_{i}\right)=\left\|\mathbf{F}_{\mathcal{P}_{1}}^{*} \mathbf{F}_{\mathcal{P}_{2}}\right\|_{F}^{2} .
$$

Multiplying out (13) and using (14), we can see that

$$
d\left(\mathbf{F}_{\mathcal{P}_{1}}, \mathbf{F}_{\mathcal{P}_{2}}\right)=\sqrt{M-\left\|\mathbf{F}_{\mathcal{P}_{1}}^{*} \mathbf{F}_{\mathcal{P}_{2}}\right\|_{F}^{2}}=\|\sin (\underline{\phi})\|_{2}
$$

where $\sin (\underline{\phi})=\left[\sin \left(\phi_{1}\right) \cdots \sin \left(\phi_{M}\right)\right]^{T}$.

\subsection{Grassmannian Interpolator}

This different view will make the problem one of obtaining reliable subspace knowledge at the transmitter. Thus, we must design $\mathbf{F}_{n}$ for all subcarriers assuming that the transmitter has knowledge of $\mathbf{F}^{(1)}, \mathbf{F}^{(2)}, \ldots, \mathbf{F}^{(K)}$ for the pilot precoders.

It has been shown in [15], [35] that precoder performance is dependent on the subspace distance $d^{2}\left(\mathbf{F}_{n}, \mathbf{F}_{n, \text { opt }}\right)$ where $\mathbf{F}_{n, \text { opt }}$ is the optimal precoder for subcarrier $n$ assuming that $\mathbf{H}_{n}$ is perfectly known to the transmitter. Thus, we would like the precoder subspace used for signal transmission at each subcarrier to be "close" to the optimal subspace assuming perfect transmitter knowledge of all subcarrier channels. Therefore, we would like to reconstruct the subspaces (and corresponding precoder matrices) for all subcarriers using the pilot precoder matrices.

To solve this subspace precoding problem, we can formulate a weighted least squares problem with the 
cost function

$$
\mathbf{F}_{n}=\underset{\mathbf{F} \in \mathcal{U}\left(M_{t}, M\right)}{\operatorname{argmin}} \sum_{k=0}^{K-1} \alpha_{n}^{2}(k) d^{2}\left(\mathbf{F}, \mathbf{F}^{(k)}\right)
$$

where $\alpha_{n}(1), \cdots, \alpha_{n}(K)$ are real valued weights for the estimation at subcarrier $n$. This weighted least squares solution will provide a good approximation to the optimal precoder without requiring any channel knowledge besides the pilot precoder matrices.

The solution we will develop for interpolating will be assuming that a weight set is determined offline. Notice that an arbitrary subcarrier number $n$ can always be uniquely written as $n=k \frac{N}{K}+l$ where $l=$ $\bmod (n, N / K)$. Using this notation, the weights for a traditional linear interpolator can be written as [1], [6]

$$
\alpha_{n}(k)= \begin{cases}\frac{N-K \bmod (n, N / K)}{N} & \text { if } k=\lfloor n K / N\rfloor \\ \frac{K \bmod (n, N / K)}{N} & \text { if } k=\lfloor n K / N\rfloor+1 \\ 0 & \text { otherwise. }\end{cases}
$$

Alternatively, second-order interpolation could be used [36]

$$
\alpha_{n}(k)= \begin{cases}\frac{\beta(\beta-1)}{2} & \text { if } k=\lfloor n K / N\rfloor-1 \\ -(\beta-1)(\beta+1) & \text { if } k=\lfloor n K / N\rfloor \\ \frac{\beta(\beta+1)}{2} & \text { if } k=\lfloor n K / N\rfloor+1 \\ 0 & \text { otherwise }\end{cases}
$$

where $\beta=\frac{K \bmod (n, N / K)}{N}$.

Given $\left\{\alpha_{n}(k)\right\}$ for all subcarriers, we will now solve (16). Note that

$$
\begin{aligned}
\sum_{k=0}^{K-1} \alpha_{n}^{2}(k) d^{2}\left(\mathbf{F}, \mathbf{F}^{(k)}\right) & =\sum_{k=0}^{K-1} \alpha_{n}^{2}(k)\left(M-\left\|\mathbf{F}^{(k)^{*}} \mathbf{F}\right\|_{F}^{2}\right) \\
& =\left(M \sum_{k=0}^{K-1} \alpha_{n}^{2}(k)\right)-\sum_{k=0}^{K-1} \alpha_{n}^{2}(k)\left\|\mathbf{F}^{(k)^{*}} \mathbf{F}\right\|_{F}^{2}
\end{aligned}
$$

The first term in (17) does not have any effect on the optimization in (16). Therefore, the problem can be reformulated as

$$
\mathbf{F}_{n}=\underset{\mathbf{F} \in \mathcal{U}\left(M_{t}, M\right)}{\operatorname{argmax}} \sum_{k=0}^{K-1} \alpha_{n}^{2}(k)\left\|\mathbf{F}^{(k)^{*}} \mathbf{F}\right\|_{F}^{2}
$$

Let

$$
\mathbf{W}_{n}=\left[\begin{array}{llll}
\alpha_{n}(0) \mathbf{F}^{(0)} & \alpha_{n}(1) \mathbf{F}^{(1)} & \ldots & \alpha_{n}(K-1) \mathbf{F}^{(K-1)}
\end{array}\right] .
$$

This weighted matrix allows (18) to be rewritten as

$$
\mathbf{F}_{n}=\underset{\mathbf{F} \in \mathcal{U}\left(M_{t}, M\right)}{\operatorname{argmax}}\left\|\mathbf{W}_{n}^{*} \mathbf{F}\right\|_{F}^{2}
$$


Consider the singular value decomposition (SVD) of $\mathbf{W}_{n}$ denoted by

$$
\mathbf{W}_{n}=\mathbf{U}_{L} \boldsymbol{\Sigma} \mathbf{U}_{R}^{*}
$$

where $\mathbf{U}_{L} \in \mathcal{U}\left(M_{t}, M_{t}\right), \mathbf{U}_{R} \in \mathcal{U}(K M, K M)$, and $\boldsymbol{\Sigma}$ is an $M_{t} \times K M$ diagonal matrix with the diagonal element at position $(i, i)$ equal to the $i^{\text {th }}$ largest singular value. It follows that [31]

$$
\begin{aligned}
\left\|\mathbf{W}_{n}^{*} \mathbf{F}\right\|_{F}^{2} & =\left\|\boldsymbol{\Sigma}^{T} \mathbf{U}_{L}^{*} \mathbf{F}\right\|_{F}^{2} \\
& \leq \sum_{i=1}^{M} \lambda_{i}\left\{\mathbf{F}^{*} \mathbf{U}_{L} \boldsymbol{\Sigma} \boldsymbol{\Sigma}^{T} \mathbf{U}_{L}^{*} \mathbf{F}\right\} \\
& =\|\overline{\boldsymbol{\Sigma}}\|_{F}^{2}
\end{aligned}
$$

where $\lambda_{i}$ is the $i^{\text {th }}$ largest singular value and $\bar{\Sigma}$ is the matrix consisting of the first $M$ columns of $\boldsymbol{\Sigma}$. The upper bound is achieved when $\mathbf{F}=\overline{\mathbf{U}}_{L}$ where $\overline{\mathbf{U}}_{L}$ is the matrix formed from the $M$ left singular vectors of $\mathbf{W}_{n}$ corresponding to the $M$ largest singular values.

This solution is equivalent to signaling on the $M$ largest principal components in the weighted pilot precoder matrix $\mathbf{W}_{n}$. The idea is that the weights $\left\{\alpha_{n}(k)\right\}$ adjust the effect of the subspace directionality in $\mathbf{F}^{(k)}$ on the precoder at subcarrier $n$. In the case when $L=1$ (i.e., flat fading), the optimization in (20) yields that all tones will signal using the same precoder as would be expected.

\section{Numerical Performance Analysis}

In this section, we compare the performance of the proposed Grassmannian interpolator using linear interpolation weights with the modified spherical (MS) interpolators in [19]-[21] and "brick-wall" type precoding as in [37]. For the beamforming scenario, the MS interpolator is

$$
\mathbf{F}_{n}=\frac{\alpha_{n}(k) \mathbf{F}^{(k)}+\alpha_{n}(k+1) e^{j \theta_{k}} \mathbf{F}^{(k+1)}}{\left\|\alpha_{n}(k) \mathbf{F}^{(k)}+\alpha_{n}(k+1) e^{j \theta_{k}} \mathbf{F}^{(k+1)}\right\|_{2}}
$$

where $\theta_{k}$ is a phase parameter that maximizes the effective channel gain of the subcarrier furthest from the interpolating precoders. It is chosen from a finite sized codebook $\Theta$ and is calculated by

$$
\theta_{k}=\underset{\Theta}{\operatorname{argmax}}\left\|\mathbf{H}_{n} \mathbf{F}_{n}\right\|_{2}^{2} \quad n=(k+1 / 2) N / K
$$


The weights $\alpha_{n}(k)$ are for those of linear interpolation. The modified spherical interpolator can be thought of as a variant of a normalized linear interpolator with an extra parameter $e^{j \theta_{k}}$. For the spatial multiplexing case, a similar expression to (22) is obtained in [21] where the $e^{j \theta_{k}}$ parameter is replaced by a rotation matrix Q followed by an appropriate orthonormalization. Alternatively, a simpler precoding scheme is to reuse the pilot precoders for the neighboring subcarriers resulting in a "brick-wall" type arrangement of the precoding matrices with

$$
\mathbf{F}_{n}=\mathbf{F}^{(k)} \quad n=(k-1 / 2) N / K \cdots(k+1 / 2) N / K .
$$

This is computationally the simplest scheme possible.

Simulations are done for a MIMO-OFDM system using QPSK with 128 subcarriers $(N=128)$ and 16 pilot tones $(K=16)$. We assume that the discrete-time channel impulse response has eight taps between each transmit and receive antennas pair with a uniform power delay profile and i.i.d. complex Gaussian distribution as in (1). We also assume that the feedback channel has no delay and no transmission error and that the receiver has perfect channel knowledge. This will allow us to isolate the effect of subspace interpolation.

Fig. 1 shows the performance of a $2 \times 4\left(M_{t} \times M_{r}\right)$ system with beamforming at the transmitter and maximum ratio combining at the receiver. For the MS interpolators, $\theta$ is uniformly quantized to 2 bits. Therefore, with MS interpolation, using a codebook size $\operatorname{card}(\mathcal{F})=2$ or $\operatorname{card}(\mathcal{F})=4$ for the beamforming vectors, a total of 48 bits $(K+2 K)$ or 64 bits $(2 K+2 K)$ of feedback information are required per OFDM symbol. The Grassmannian interpolator used a codebook size $\operatorname{card}(\mathcal{F})=8$ which requires 48 bits $(3 K)$ of feedback information. The benchmark for comparison is where the indices from a codebook size $\operatorname{card}(\mathcal{F})=16$ are fed back for all beamforming vectors. This requires a total of 512 bits of feedback information. The Grassmann interpolator using 48 bits of feedback has the same performance as that of the MS interpolator with 64 bits and "brick-wall" scheme with 80 bits. The performance gains of the Grassmann interpolator over the MS interpolator are also presented in a coded system. Fig. 2 shows the bit error rate (BER) curves when using a rate $1 / 2$ convolutional code in the above system with generator polynomials $g_{0}=133_{8}$ and 
$g_{1}=171_{8}$. The interleaver specified in the industry standard [38] is used on a per MIMO-OFDM symbol basis and soft decision decoding is done with the Viterbi algorithm.

It is also of interest to compare the performance of the above system in a multi-user scenario. In this model, up to $P$ users are allowed to transmit in each of the $N$ subcarriers. The received signal in the $n^{t h}$ subcarrier for the $p^{\text {th }}$ user can be written as

$$
\mathbf{Y}_{n, p}=\mathbf{H}_{n, p} \mathbf{X}_{n, p}+\left(\sum_{\mu=1, \mu \neq p}^{P} \mathbf{H}_{n, \mu} \mathbf{X}_{n, \mu}\right)+\mathbf{N}_{n, p}
$$

If the channel model for each of the users follows (1) then the interference term in the brackets above for the $p^{\text {th }}$ user is Gaussian. In Fig. 3 we plot the interpolator performance with the signal to interferer power fixed at 5dB. The Grassmann interpolator using 48 bits of feedback has a performance comparable to the MS interpolator using 64 bits of feedback.

In Fig. 4 the performance of the different interpolators are compared for a two substream precoded spatial multiplexing $4 \times 2$ system. We plot the vector symbol error probability when using zero-forcing (ZF) receivers. The precoding matrices are found using the minimum singular value criterion [16] and are selected from codebooks generated using methods in [39]. For the MS interpolator, the unitary rotation matrix $\mathbf{Q}$ is chosen from a codebook size $\operatorname{card}(\mathcal{Q})=4$. With the precoding matrices chosen from a codebook size $\operatorname{card}(\mathcal{F})=4$, the MS scheme requires 64 bits $(2 K+2 K)$ of feedback. The corresponding Grassmannian interpolator with $\operatorname{card}(\mathcal{F})=16$ also uses 64 bits $(4 K)$ of feedback. The ideal case for comparison is where the indices of the precoder matrices for all subcarriers are sent back from a codebook size of $\operatorname{card}(\mathcal{F})=64$ corresponding to 768 bits $(6 N)$ of feedback. At a vector symbol error rate (VSER) of $10^{-1}$ the Grassmannian interpolator outperforms the MS interpolator by $0.55 \mathrm{~dB}$ and is $1.25 \mathrm{~dB}$ away from the ideal case.

In Fig. 5 we plot the performance of the precoding schemes when there is channel estimation error in the above spatial multiplexing system. The receiver is assumed to estimate the channel matrix for the $n^{t h}$ subcarrier as being

$$
\mathbf{H}_{n, \text { est }}=\alpha \mathbf{H}_{n}+\sqrt{1-\alpha^{2}} \mathbf{H}_{n, \text { error }}
$$


where the entries of $\mathbf{H}_{n, \text { error }}$ are independent and distributed according to $\mathcal{C N}(0,1)$. The precoders for the pilots are designed as a function of $\mathbf{H}_{n, e s t}$. As can be seen, the degradation in performance due to increasing channel estimation error is less with the Grassmann interpolator. When $\alpha^{2}=0.9$, the performance of the Grassmann interpolator is as good as that of the MS interpolator with perfect channel knowledge.

Finally, we compare the computational complexities for the Grassmann and MS interpolators for precoded spatial multiplexing systems. For the Grassmann interpolator an $O\left(K \cdot 2^{B}\right)$ search is first required at the receiver to determine the best precoder matrices for the pilots. The computational cost for the transmitter to compute the precoder matrices for the non-pilot subcarriers is $(N-K) \cdot O\left(M_{t}^{3}\right)$. In the MS interpolator the receiver has to do an $O\left(K \cdot\left(2^{B}+2^{B_{1}}\right)\right)$ search where $B_{1}=\log _{2}(\operatorname{card}(\mathcal{Q}))$ to determine the precoder matrices for the pilots. The computational cost at the transmitter to compute the precoder matrices for the non-pilot subcarriers is $(N-K) \cdot O\left(M^{3}\right)$.

\section{Conclusions}

In this correspondence, we proposed a method for performing precoding with pilot-based feedback. The idea is to formulate the per subcarrier precoder design problem using pilot tone feedback as a weighted least squared subspace distance problem. With this formulation, we show the problem can be easily solved using principle component techniques. The algorithm can use any interpolation weighting scheme.

\section{References}

[1] L. J. Cimini, Jr., "Analysis and simulation of a digital mobile channel using orthogonal frequency division multiplexing," IEEE Trans. Commun., vol. 33, pp. 665-675, July 1985.

[2] Y. G. Li, L. J. Cimini, Jr., and N. R. Sollenberger, "Robust channel estimation for OFDM systems with rapid dispersive fading channels," IEEE Trans. Commun., vol. 46, pp. 902-915, July 1998.

[3] J. Rinne and M. Renfors, "Pilot spacing in orthogonal frequency division multiplexing systems on practical channels," IEEE Trans. Consumer Elec., vol. 42, pp. 959-962, Nov. 1996.

[4] M.-H. Hsieh and C.-H. Wei, "Channel estimation for OFDM systems based on comb-type pilot arrangement in frequency selective fading channels," IEEE Trans. Consumer Elec., vol. 44, pp. 217-225, Feb. 1998.

[5] W.-G. Song and J.-T. Lim, "Pilot-symbol aided channel estimation for OFDM with fast fading channels," IEEE Trans. Broadcasting, vol. 49, pp. 398-402, Dec. 2003.

[6] S. Coleri, M. Ergen, A. Puri, and A. Bahai, "Channel estimation techniques based on pilot arrangement in OFDM systems," IEEE Trans. Broadcasting, vol. 48, pp. 223-229, Sept. 2002.

[7] A. Scaglione, P. Stoica, S. Barbarossa, G. B. Giannakis, and H. Sampath, "Optimal designs for spacetime linear precoders and decoders," IEEE Trans. Sig. Proc., vol. 50, pp. 1051-1064, May 2002. 
[8] H. Sampath, P. Stoica, and A. Paulraj, "Generalized precoder and decoder design for MIMO channels using the weighted MMSE criterion," IEEE Trans. Commun., vol. 49, pp. 2198-2206, Dec. 2001.

[9] G. Jöngren, M. Skoglund, and B. Ottersten, "Combining beamforming and orthogonal space-time block coding," IEEE Trans. Info. Th., vol. 48, pp. 611-627, March 2002.

[10] A. Narula, M. J. Lopez, M. D. Trott, and G. W. Wornell, "Efficient use of side information in mulitipleantenna data transmission over fading channels," IEEE Jour. Select. Areas in Commun., vol. 16, pp. 1423-1436, Oct. 1998.

[11] E. G. Larsson, G. Ganesan, P. Stoica, and W.-H. Wong, "On the performance of orthogonal space-time block coding with quantized feedback," IEEE Commun. Lett., vol. 6, pp. 487-489, Nov. 2002.

[12] D. J. Love, R. W. Heath Jr., and T. Strohmer, "Grassmannian beamforming for multiple-input multipleoutput wireless systems," IEEE Trans. Info. Th., vol. 49, pp. 2735-2747, Oct. 2003.

[13] K. K. Mukkavilli, A. Sabharwal, E. Erkip, and B. Aazhang, "On beamforming with finite rate feedback in multiple antenna systems," IEEE Trans. Info. Th., vol. 49, pp. 2562-2579, Oct. 2003.

[14] G. Jöngren and M. Skoglund, "Quantized feedback information in orthogonal space-time block coding," IEEE Trans. Info. Th., vol. 50, pp. 2473-2486, Oct. 2004.

[15] D. J. Love and R. W. Heath, Jr., "Limited feedback unitary precoding for orthogonal space-time block codes," IEEE Trans. Sig. Proc., vol. 53, pp. 64-73, Jan. 2005.

[16] D. J. Love and R. W. Heath, Jr., "Limited feedback unitary precoding for spatial multiplexing systems," IEEE Trans. Info. Th., July 2005.

[17] W. Santipach and M. L. Honig, "Asymptotic performance of MIMO wireless channels with limited feedback," in Proc. IEEE Mil. Comm. Conf., vol. 1, pp. 141-146, Oct. 2003.

[18] S. Zhou, Z. Wang, and G. B. Giannakis, "Performance analysis for transmit-beamforming with finiterate feedback," in Proc. Conf. on Inform. Sciences and Systems, March 2004.

[19] J. Choi and R. W. Heath, Jr., "Interpolation based transmit beamforming for MIMO-OFDM with limited feedback," IEEE Trans. Sig. Proc., vol. 53, pp. 4125-4135, Nov. 2005.

[20] J. Choi and R. W. Heath, Jr., "Interpolation based transmit beamforming for MIMO-OFDM with limited feedback," in Proc. IEEE Int. Conf. on Commun., vol. 1, pp. 249-253, June 2004.

[21] J. Choi and R. W. Heath, Jr., "Interpolation based unitary precoding for spatial multiplexing MIMOOFDM with limited feedback," in Proc. IEEE Glob. Telecom. Conf., vol. 1, pp. 214-218, Nov.-Dec. 2004.

[22] A. Paulraj, R. Nabar, and D. Gore, Introduction to Space-Time Wireless Communications. New York: Cambridge University Press, 2003.

[23] G. J. Foschini, "Layered space-time architecture for wireless communication in a fading environment when using multiple antennas," Bell Labs Technical Journal, vol. 1, pp. 41-59, Autumn 1996.

[24] S. M. Alamouti, "A simple transmit diversity technique for wireless communications," IEEE Jour. Select. Areas in Commun., vol. 16, pp. 1451-1458, Oct. 1998.

[25] V. Tarokh, H. Jafarkhani, and A. R. Calderbank, "Space-time block codes from orthogonal designs," IEEE Trans. Info. Th., vol. 45, pp. 1456-1467, July 1999.

[26] D. A. Gore and A. J. Paulraj, "MIMO antenna subset selection with space-time coding," IEEE Trans. Sig. Proc., vol. 50, pp. 2580-2588, Oct. 2002.

[27] R. W. Heath Jr., S. Sandhu, and A. Paulraj, "Antenna selection for spatial multiplexing systems with linear receivers," IEEE Commun. Lett., vol. 5, pp. 142-144, April 2001.

[28] R. S. Blum and J. H. Winters, "On optimum MIMO with antenna selection," IEEE Commun. Lett., vol. 6, pp. 322-324, Aug. 2002.

[29] P. A. Dighe, R. K. Mallik, and S. S. Jamuar, "Analysis of transmit-receive diversity in Rayleigh fading," IEEE Trans. Commun., vol. 51, pp. 694-703, April 2003.

[30] R. Narasimhan, "Spatial multiplexing with transmit antenna and constellation selection for correlated MIMO fading channels," IEEE Trans. Sig. Proc., vol. 51, pp. 2829-2838, Nov. 2003.

[31] R. A. Horn and C. R. Johnson, Matrix Analysis. New York: Cambridge University Press, March 2001.

[32] E. G. Larsson and P. Stoica, Space-Time Block Coding for Wireless Communications. New York: Cambridge University Press, 2003. 
[33] S. Sandhu, R. U. Nabar, D. A. Gore, and A. Paulraj, "Near-optimal selection of transmit antennas for a MIMO channel based on Shannon capacity," in Proc. 34th Asilomar Conf. on Sig., Sys., and Comp., vol. 1, Nov. 2000.

[34] A. Barg and D. Y. Nogin, "Bounds on packings of spheres in the Grassmann manifold," IEEE Trans. Info. Th., vol. 48, pp. 2450-2454, Sept. 2002.

[35] D. J. Love and R. W. Heath, Jr., "Limited feedback precoding for spatial multiplexing systems," in Proc. IEEE Glob. Telecom. Conf., vol. 4, pp. 1857-1861, Dec. 2003.

[36] G.-S. Liu and C.-H. Wei, "A new variable fractional sample delay filter with nonlinear interpolation," IEEE Transactions on Circuits and Systems-Part II: Analog and Digital Signal Processing, vol. 39, pp. 123-126, Feb 1992.

[37] R. Grunheid, E. Bolinth, and H. Rohling, "A block-wise loading algorithm for the adaptive modulation technique in OFDM systems," in Proc. IEEE Veh. Technol. Conf., vol. 2, pp. 948-951, Oct. 2001.

[38] IEEE Std 802.11a, Part11: Wireless LAN medium access control (MAC) and physical layer (PHY) specifications-High speed physical layer in the 5GHz band, LAN/MAN Standards Committee, 1999.

[39] B. M. Hochwald, T. L. Marzetta, T. J. Richardson, W. Sweldens, and R. Urbanke, "Systematic design of unitary space-time constellations," IEEE Trans. Info. Th., vol. 46, pp. 1962-1973, Sept. 2000.

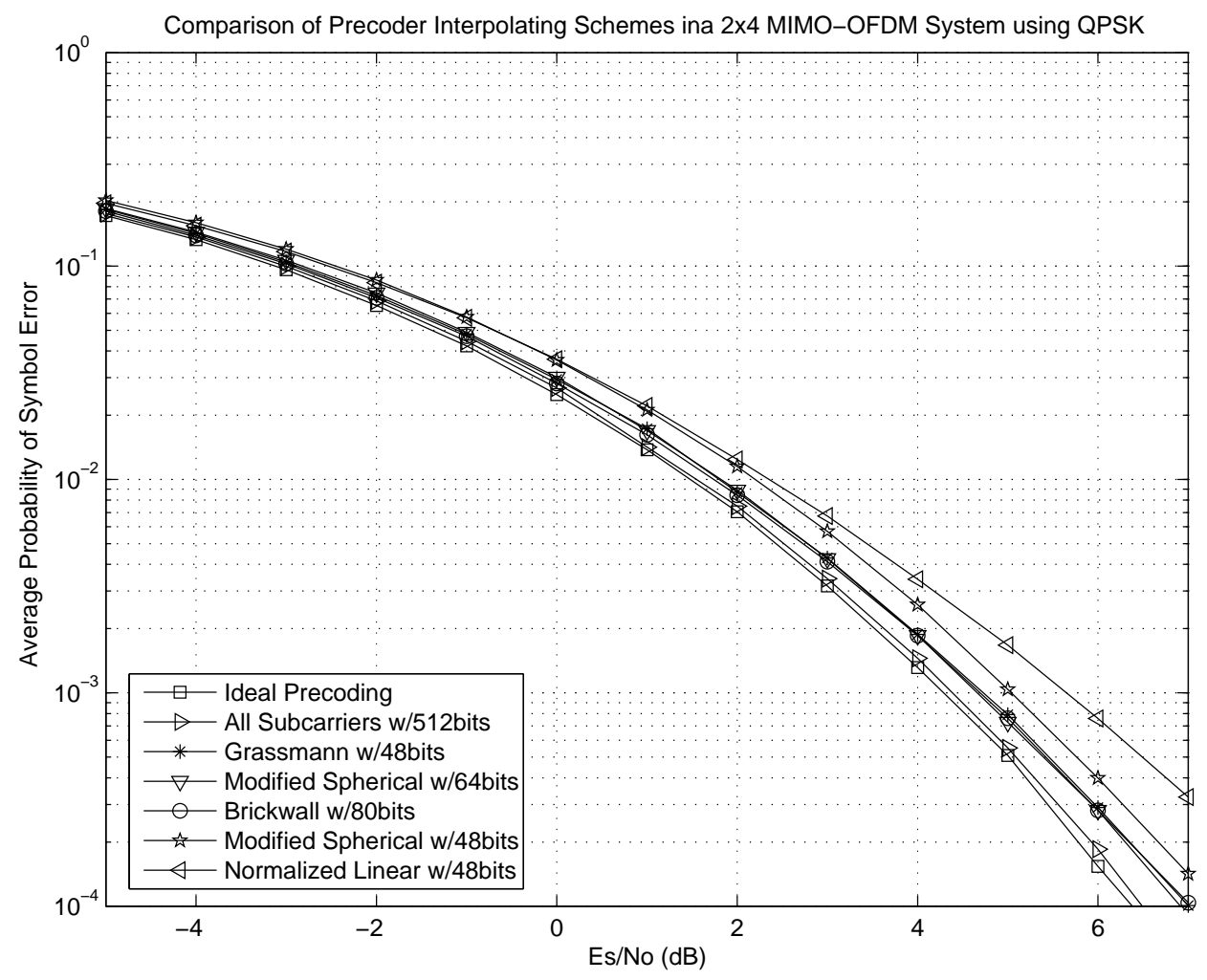

Figure 1: Probability of symbol error comparison for different precoder interpolation schemes in a $2 \times 4$ beamforming MIMO-OFDM system using QPSK. 


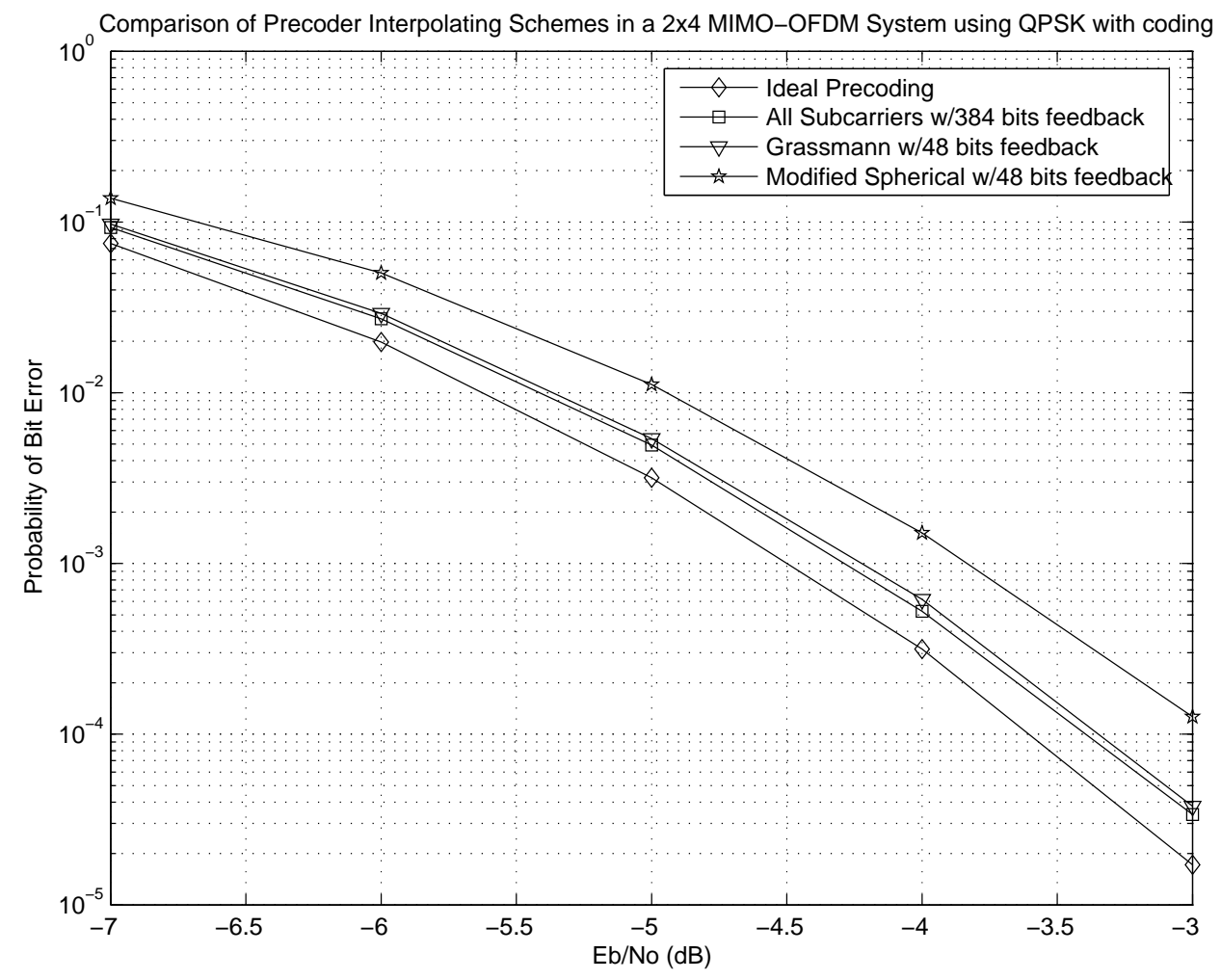

Figure 2: Bit error rate comparison for different precoder interpolation schemes in a $2 \times 4$ beamforming MIMO-OFDM system using QPSK with coding.

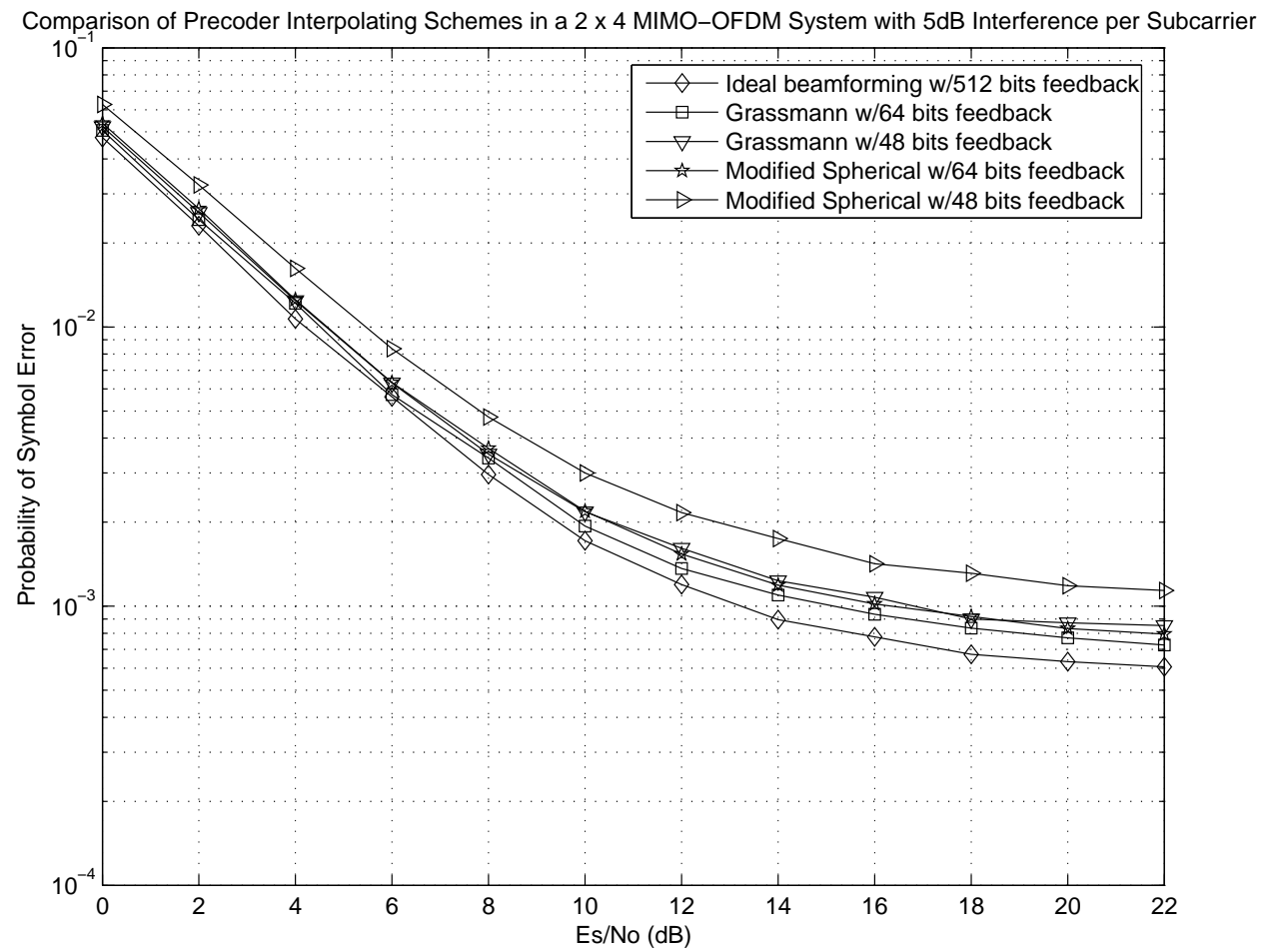

Figure 3: Probability of symbol error comparison for different precoder interpolation schemes in a $2 \times 4$ beamforming MIMO-OFDM system using QPSK. The signal-to-interference power was fixed at $5 \mathrm{~dB}$ 


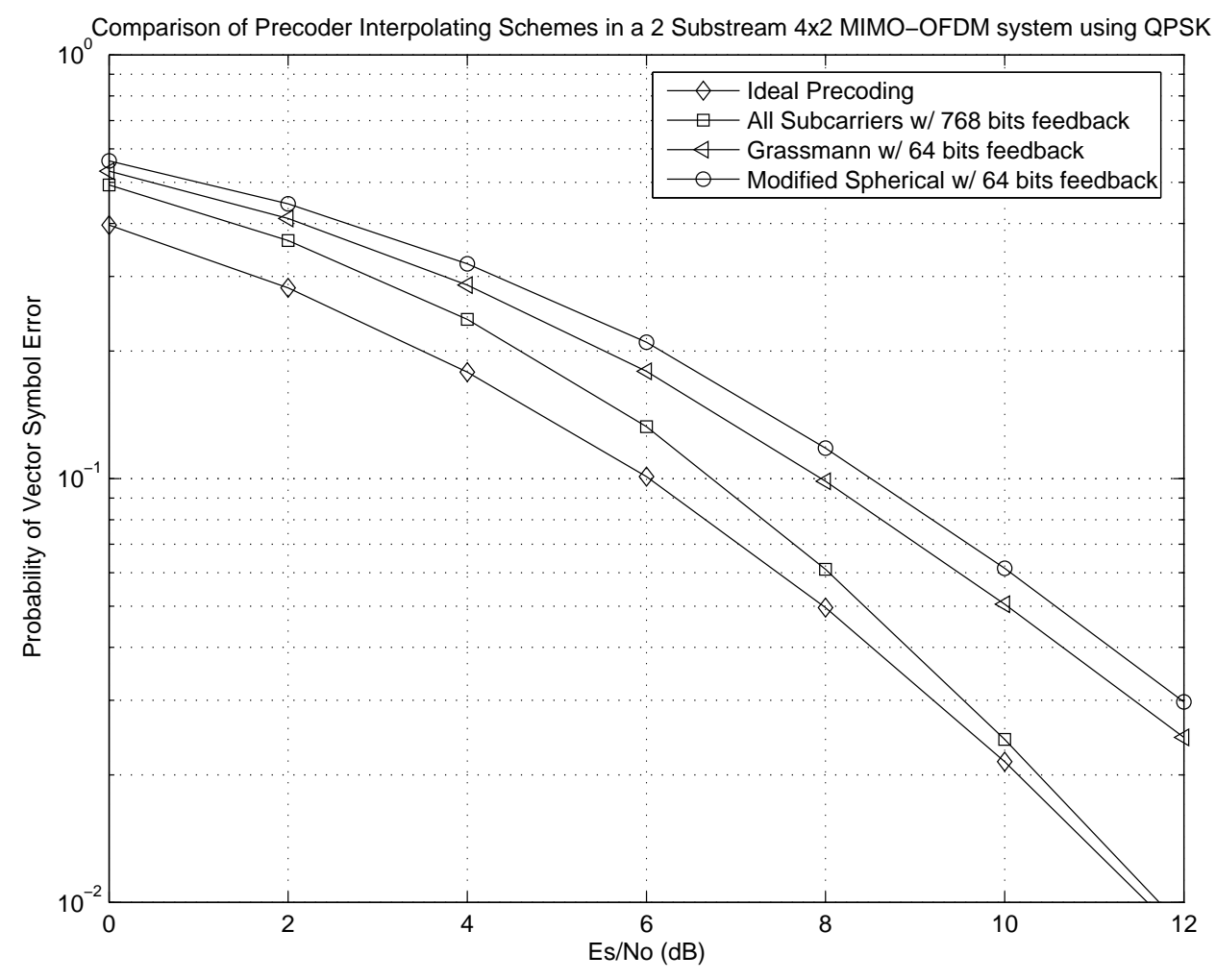

Figure 4: Probability of symbol vector error for different precoder interpolation schemes in a two substream $4 \times 2$ MIMO-OFDM system using QPSK with a zero-forcing receiver.

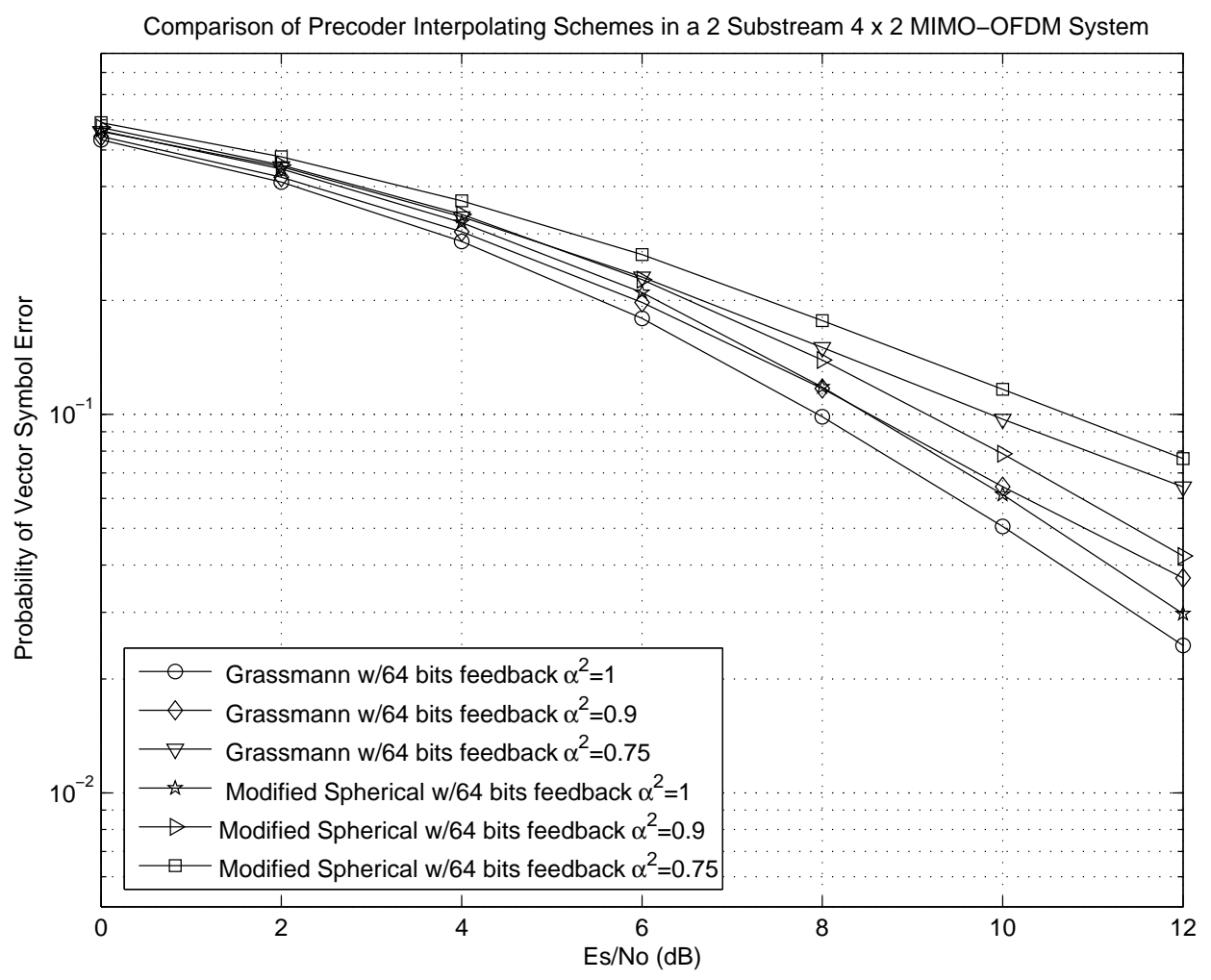

Figure 5: Probability of vector symbol error for different precoder interpolation schemes with channel estimation error in a two substream $4 \times 2$ MIMO-OFDM system using QPSK with a zero-forcing receiver. 